\title{
Many Aspects of Magnetic Fields in Neutron Stars
}

\author{
Rodrigo Negreiros $^{1, *}$, Cristian Bernal ${ }^{2}$, Veronica Dexheimer ${ }^{3}$ (i) and Orlenys Troconis ${ }^{1}$ \\ 1 Instituto de Física, Universidade Federal Fluminense, Praia Vermelha 24210-346, Brazil; \\ troconisorlenys@gmail.com \\ 2 Instituto de Matemática, Estatísca e Física, Universidade Federal de Rio Grande, de Rio Grande 96200-970, \\ Brazil; cgbernal@furg.br \\ 3 Department of Physics, Kent State University, Kent, OH 44240, USA; vdexheim@kent.edu \\ * Correspondence: rnegreiros@id.uff.br
}

Received: 11 December 2017; Accepted: 7 February 2018; Published: 26 February 2018

\begin{abstract}
In this work, we explore different aspects in which strong magnetic fields play a role in the composition, structure and evolution of neutron stars. More specifically, we discuss (i) how strong magnetic fields change the equation of state of dense matter, alter its composition, and create anisotropies, (ii) how they change the structure of neutron stars (such mass and radius) and the formalism necessary to calculate those changes, and (iii) how they can affect neutron stars' evolution. In particular, we focus on how a time-dependent magnetic field modifies the cooling of a special group known as $\mathrm{X}$-ray dim neutron stars.
\end{abstract}

Keywords: neutron stars; stellar magnetic field; stellar structure; stellar evolution

\section{Introduction}

Since the detection of a soft gamma repeater in 1979 and an anomalous X-ray pulsar in 1981, people became interested in neutrons stars that could be powered by their strong magnetic field. In 1992 and 1993, Duncan and Thompson proposed the magnetar model [1,2] and, since then, approximately 30 Soft-Gamma Ray Repeaters (SGRs) and Anomalous X-Ray Pulsars (AXP's) have been observed [3] with thousands of them being expected to exist in our galaxy. Today, these two kinds of objects are understood as being one class of objects, magnetars [4-6]. In recent years, several measurements have shed new light on the strength of magnetic fields on the surface and in the interior of neutron stars. While measurements using anharmonic precession of star spin down have estimated surface magnetic fields to be on the magnitude of $10^{15} \mathrm{G}$ for the sources 1E 1048.1-5937 and 1E 2259+586 [7], data for slow phase modulations in star hard X-ray pulsations (interpreted as free precession) suggest internal magnetic fields to be on the magnitude of $10^{16} \mathrm{G}$ for the source $4 \mathrm{U} 0142+61$ [8]. Together, these estimates have motivated a large amount of research on the issue of how magnetic fields modify the physics of neutron stars.

In addition to the objects discussed above, magnetic fields are a key aspect in the evolution of neutron stars. Particularly interesting are the objects known as X-ray dim neutron stars (XDINS). These objects show bright soft X-ray emission, exhibit blackbody-like spectra, and have high temperatures with respect to their spin-down age. In fact, one of the most puzzling aspects of the XDINS is that their spin-down properties (period and period derivative) indicate old ages (the so-called spin-down age), which is unexpected, considering their high observed temperatures.

Motivated by the scenarios discussed above, in this work we will discuss the most prominent aspects of magnetic field for the composition, structure and evolution of neutron stars. Initially we will focus on the microscopic aspects and, in particular, how magnetic fields may change the equation of state of dense matter through Landau quantization, which generates pressure anisotropies, and affect the particle composition. After that, we will concentrate on the macroscopic aspects, in particular on 
the question of how strong magnetic fields change the structure of neutron stars (such mass and radius), we will also briefly discuss the formalism which is necessary to take care of the anisotropies generated by the magnetic field. Finally, we are going to discuss how magnetic fields may affect neutron stars' temporal evolution, particularly, we expand on the idea that the spin-down age may mask the true age of the neutron star if its magnetic field experiences a non-canonical behavior, namely if it is evolving with time. We note here that in this work we will address these three fronts (microscopic, macroscopic and evolutionary) in a up to some extent independent manner, given the significant challenge that is to treat all of them on the same footing. Our ultimate goal is to build a framework in which such treatment may be viable, thus in here we lay the ground work for such investigation and present our perspective to performing such self-consistent overarching study.

\section{Microscopic Aspects}

The inclusion of an external magnetic field in one direction generates the quantization of energy levels in the directions perpendicular to the magnetic field, what is referred to as Landau levels. These levels are related to the particles' angular momentum quantum number, but also charge and spin. The levels are degenerated, except for the zeroth level. This formalism was originally derived for the non-relativistic case by Landau [9]. In the case of finite temperature, Landau levels are summed from zero to infinity but, in the special case of zero temperature, there is a maximum Landau level beyond which the particles' Fermi momenta in the direction of the field become negative. As a consequence, the 3-dimensional integrals for the thermodynamical quantities become 1-dimensional but summed over all the possible levels. On one hand, very strong magnetic fields restrict the summations to the lower Landau levels, whereas very week fields require summations over many levels, becoming at some point continuous. In this case, the integrals become once more 3-dimensional. This formalism, when applied to neutron-star matter with homogeneous magnetic fields [10-14] generates softer equations of state, as the enhancement of charged particles also turns the system more isospin symmetric. Of course, a possible suppression of hyperons by the magnetic field has exactly the opposite effect on the equation of state (as hyperons turn the equation of state softer) and phase transitions to quark matter can have different effects [15].

In addition, the energy momentum tensor becomes anisotropic, as the pressure in the directions parallel and perpendicular to the magnetic field become distinct. This issue first raised by Canuto [16,17] happens due to an extra contribution from the fermions, which move in their quantized orbits perpendicular to the external magnetic field. This issue was recently revisited in recent publications [18-20]. Another effect created by the magnetic field concerns its interaction with the fermions' spin, the anomalous magnetic moment $[18,21]$. In this case, the magnetic field turns the equation of state of hadronic matter stiffer, due to a modification of the amount of particles with different spins (polarization) [22,23]. For quarks, the efficiency of anomalous magnetic moment has been estimated to be very small [24,25].

As it is going to be shown in the next section, the effects discussed above concerning stiffness and anisotropy of the equation of state and particle population do not have a substantial effect on macroscopic properties of neutrons stars, such as stellar masses [26,27]. On the other hand strong magnetic fields decrease substantially the central density of neutron star. In this case, the particle population inside a neutron star can be substantially modified, as shown in Figure 1. As explained in detail in Ref. [27], very strong magnetic fields (but still realistic) can suppress not only a phase transition to quark matter, but also the appearance of hyperons (bottom panels of Figure 1). But, interestingly, as the magnetic field strength throughout the star decays over time (more details about magnetic field temporal evolution in Section 4), an increase in central density or chemical potential allows the appearance of hyperons and then quarks, as shown in the top panels of Figure 1. These calculations were performed by solving numerically the Einstein-Maxwell equations at fixed baryon mass $M_{B}=2.2 \mathrm{M}_{\odot}$ using the chiral mean field (CMF) model [28]. 


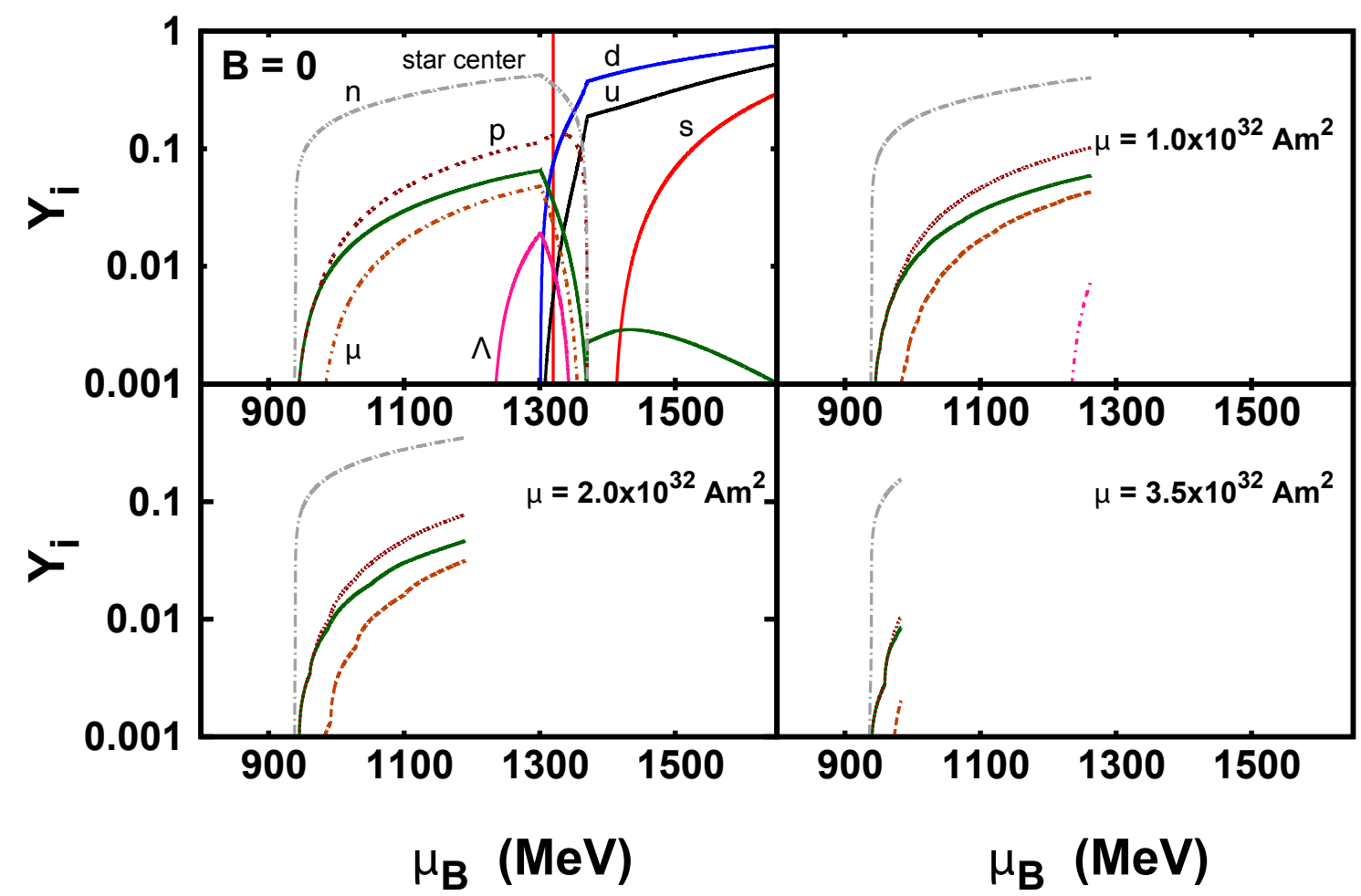

Figure 1. Particle population as a function of the baryon chemical potential calculated using the CMF model shown for different dipole magnetic moments $\mu$. All figures represent equilibrium sequences obtained from the numerical solution of he Einstein-Maxwell equations at fixed baryon mass. For the non-magnetized case, the vertical red curve represents the chemical potential reached at the center of the star, namely, $1320 \mathrm{MeV}$.

Another relevant question is how much does the magnetic field vary inside neutron stars. As already pointed out by Menezes et al. in Ref. [29], ad hoc formulas for magnetic field profiles in neutron stars do not fulfill Maxwell's equations (more specifically, Gauss' law) and, therefore, are incorrect. The Ref. [30] showed that strong magnetic fields inside magnetic neutron stars increase quadratically with baryon chemical potential in the polar direction and not by more than one order of magnitude when solving the Einstein-Maxwell's equations. This study was performed independently for several different equations of state but did not provide profiles for the magnetic field strength in the stellar equatorial direction, as those are much more complicated.

\section{Macroscopic Aspects}

Following the microscopic discussion we now address how the magnetic field may alter the macroscopic properties of a neutron star. For that we must fully describe magnetic field in a general relativistic environment as well as the deviation from spherical symmetry that are associated with it. Since the seminal paper by Oppenheimer and Snyder [31], most of the work dedicated to the problem of general relativistic sources, deal with spherically symmetric fluid distribution. In reality, the study of self gravitating compact objects one usually assumes that small deviations from spherical symmetry are likely to take place. Such small deviations are not appropriate for stars with strong magnetic fields where a full axially symmetric treatment is necessary to properly describe the system. The population statistics of SGRs suggest that more than $10 \%$ of neutron stars are born as magnetars, with their magnetic field decaying as they age [32-34]. Hence it seems likely that some mechanism is capable of generating large magnetic fields in nascent neutron stars.

In this section we will describe such highly magnetized neutron stars as a perfect fluid coupled with a dipolar magnetic field. First, we will discuss the theoretical aspects relevant to the magnetic field 
role on the structure of neutron stars, more specifically, we will discuss conservation equations, which will be written in terms of three quantities - whose physical interpretation will be given. Furthermore, we will also discuss calculations of highly magnetized neutron stars, whose structure have been calculated numerically. We will discuss how the presence of a strong (albeit realistic) magnetic field affects global, and potentially observational properties of neutron stars such as gravitational mass and radius.

\subsection{Formal Aspects of the Magnetic Field on the Structure of Neutron Stars}

Now we briefly discuss the formal aspects of the magnetic field in the stellar structure and gravitational equations in the context of Einstein's general relativity. We begin by considering a bound, static and axially symmetric source. The line element may be written in cylindrical coordinates as

$$
d s^{2}=-A^{2}\left(d x^{0}\right)^{2}+B^{2}\left[\left(d x^{1}\right)^{2}+\left(d x^{2}\right)^{2}\right]+D^{2}\left(d x^{3}\right)^{2},
$$

where we identify $x^{0}=t, x^{1}=\rho, x^{2}=z, x^{3}=\phi$ and $A, B, D$ are positive functions of the coordinates $\rho$ and $z$. In the Weyl spherical coordinates, the line element (1) is

$$
d s^{2}=-A^{2}(d t)^{2}+B^{2}\left[(d r)^{2}+r^{2}(d \theta)^{2}\right]+D^{2}(d \phi)^{2},
$$

where $\rho=r \sin \theta$ and $z=r \cos \theta$. We denote the coordinates as $x^{\mu}=(t, r, \theta, \phi)$, and $A(r, \theta), B(r, \theta), D(r, \theta)$ are three independent functions.

The sources of curvature in Einstein's general relativity is represented by the energy-momentum tensor. For a magnetized neutron star, we describe the system as a perfect fluid coupled to a poloidal magnetic field. The perfect fluid assumption simplifies the mathematical treatment dramatically, however, there has also been research considering spherically symmetric dissipative and anisotropic fluid distribution and some of them with analytical solutions (see for instance $[35,36]$. As mentioned at the beginning of this section, highly magnetized neutron stars should be modeled using an axially symmetric metric tensor which increases the complexity of the problem considerably.

The motivation behind the assumption of a poloidal magnetic field is that such assumption is compatible with the circularity of the space-time [37]. It is important to note, however, that nonnegligible toroidal magnetic fields are likely to exist in neutron stars, making the study considerably more complicated. The study of toroidal magnetic fields, in addition to poloidal ones is beyond the scope of this work.

Following the scenario discussed above, the energy-momentum tensor for the system is written as that of a perfect-fluid in addition to the energy-momentum tensor of the electromagnetic field,

$$
T_{\mu \nu}=T_{\mu \nu}^{P F}+T_{\mu \nu}^{E M}
$$

The perfect fluid $(\mathrm{PF})$ contribution is

$$
T_{\mu \nu}^{P F}=(\rho+P) u_{\mu} u_{v}+P g_{\mu \nu}
$$

where $\rho$ and $P$ are, respectively, the rest-frame energy density and pressure, $u^{\mu}$ is the fluid 4-velocity with $u^{\mu} u_{\mu}=-1$. The electromagnetic part (EM) in (3) is

$$
T_{\mu \nu}^{E M}=\frac{1}{4 \pi}\left(F_{\mu}{ }^{\alpha} F_{\nu \alpha}-\frac{1}{4} g_{\mu \nu} F^{\alpha \beta} F_{\alpha \beta}\right)
$$

where the Maxwell tensor $F_{\mu \nu}$ is defined in terms of the electromagnetic 4-potential $A_{\mu}$ as

$$
F_{\mu \nu}=A_{v, \mu}-A_{\mu, v}
$$


We are interested in describing a distribution without free-charge and with only poloidal magnetic field, thus the electromagnetic 4-potential is written as

$$
A_{\mu}=\left(0,0,0, A_{\phi}(r, \theta)\right)
$$

The matrix form of $F_{\mu \nu}$ is written as

$$
F_{\mu v}=\left(\begin{array}{cccc}
0 & 0 & 0 & 0 \\
0 & 0 & 0 & \frac{\partial A_{\phi}}{\partial r} \\
0 & 0 & 0 & \frac{\partial A_{\phi}}{\partial \theta} \\
0 & -\frac{\partial A_{\phi}}{\partial r} & -\frac{\partial A_{\phi}}{\partial \theta} & 0
\end{array}\right)
$$

and the electromagnetic energy-momentum tensor is

$$
T_{\nu}^{E M \mu}=\left(\begin{array}{cccc}
T^{E M 0} & 0 & 0 & 0 \\
0 & T^{E M 1} & T^{E M 1}{ }^{2} & 0 \\
0 & \frac{1}{r^{2}} T^{E M 1}{ }_{2} & -T^{E M 1} 1 & 0 \\
0 & 0 & 0 & -T_{0}^{E M 0}{ }_{0}
\end{array}\right),
$$

where the non-vanishin components, in terms of the electromagnetic 4-potential, are given by

$$
\begin{gathered}
T_{0}^{E M 0}=-\frac{1}{8 \pi} g^{\phi \phi}\left[g^{r r}\left(\frac{\partial A_{\phi}}{\partial r}\right)^{2}+g^{\theta \theta}\left(\frac{\partial A_{\phi}}{\partial \theta}\right)^{2}\right] \\
T_{1}^{E M 1}=\frac{1}{8 \pi} g^{\phi \phi}\left[g^{r r}\left(\frac{\partial A_{\phi}}{\partial r}\right)^{2}-g^{\theta \theta}\left(\frac{\partial A_{\phi}}{\partial \theta}\right)^{2}\right] \\
T_{2}^{E M 1}=\frac{1}{4 \pi} g^{r r} g^{\phi \phi}\left(\frac{\partial A_{\phi}}{\partial r}\right)\left(\frac{\partial A_{\phi}}{\partial \theta}\right) .
\end{gathered}
$$

Now, inspired by Equation (10) we define the following electromagnetic quantities

$$
\begin{aligned}
& B_{\theta}=\sqrt{g^{r r}}\left(\frac{\partial A_{\phi}}{\partial r}\right), \\
& B_{r}=\sqrt{g^{\theta \theta}}\left(\frac{\partial A_{\phi}}{\partial \theta}\right),
\end{aligned}
$$

It is important to realize that these components are not exactly the components measured by the Eulerian observer, but rather convenient definitions of electromagnetic functions that allow us to write the components of $T^{E M}$ in a more intuitive manner, as

$$
\begin{aligned}
& T_{0}^{E M 0}=-\frac{1}{8 \pi} g^{\phi \phi}\left(B_{r}^{2}+B_{\theta}^{2}\right), \\
& T_{1}^{E M 1_{1}}=-\frac{1}{8 \pi} g^{\phi \phi}\left(B_{r}^{2}-B_{\theta}^{2}\right), \\
& T_{2}^{E M 1_{2}}=\frac{1}{8 \pi} 2 g^{\phi \phi} \sqrt{\frac{g^{r r}}{g^{\theta \theta}}} B_{r} B_{\theta} .
\end{aligned}
$$


Here, if we want to fully comprehend the physical meaning of the components of the electromagnetic energy-momentum tensor, we must draw a parallel with its flat-space counter-part, given (in S.I. units) as [38]

$$
T^{E M \mu v}=\left(\begin{array}{cccc}
\frac{1}{2}\left(\epsilon_{0} E^{2}+\frac{1}{\mu_{0}} B^{2}\right) & S_{x} / c & S_{y} / c & S_{z} / c \\
S_{x} / c & -\sigma_{x x} & -\sigma_{y y} & -\sigma_{z z} \\
S_{y} / c & -\sigma_{y x} & -\sigma_{y y} & -\sigma_{y z} \\
S_{z} / c & -\sigma_{z x} & -\sigma_{z y} & -\sigma_{z z}
\end{array}\right)
$$

where $\vec{S}=\frac{1}{\mu_{0}} \vec{E} \times \vec{B}$ is the Poynting vector and the components $\sigma_{i j}$ are given by

$$
\sigma_{i j}=\epsilon_{0} E_{i} E_{j}+\frac{1}{\mu_{0}} B_{i} B_{j}-\frac{1}{2}\left(\epsilon_{0} E^{2}+\frac{1}{\mu_{0}} B^{2}\right) \delta_{i j}
$$

The first term in Equation (18) is easily identified as the electromagnetic energy density, the other terms in the diagonal, i.e., $\sigma_{x x}, \sigma_{y y}, \sigma_{z z}$ can be read as the electromagnetic pressure and the terms $\sigma_{i j}$ for $i \neq j$ represents shear stress.

Inspired in the electromagnetic energy-momentum tensor for flat space-time, we define the following quantities

$$
\begin{gathered}
W \equiv \frac{1}{8 \pi} g^{\phi \phi}\left(B_{r}^{2}+B_{\theta}^{2}\right), \\
\Pi \equiv \frac{1}{8 \pi} g^{\phi \phi}\left(B_{r}^{2}-B_{\theta}^{2}\right), \\
\sigma \equiv \frac{1}{8 \pi} 2 g^{\phi \phi} B_{r} B_{\theta} .
\end{gathered}
$$

With these definitions, the matrix form of the electromagnetic energy-momentum tensor looks like

$$
T_{\nu}^{E M \mu}=\left(\begin{array}{cccc}
-W & 0 & 0 & 0 \\
0 & -\Pi & r \sigma & 0 \\
0 & \frac{1}{r} \sigma & \Pi & 0 \\
0 & 0 & 0 & W
\end{array}\right)
$$

From Equation (23) we can extract the following properties for $T^{E M}$ : it is symmetric, traceless and the component $T^{E M 00}$ is positive definite, which are the expected properties of an electromagnetic energy-momentum tensor. One must note that Equation (23) correspond to the mixed components of the electromagnetic energy-momentum tensor, whereas the properties just defined are related to the contra-variant components.

Combining Equations (4) and (23), the matrix form of the energy-momentum tensor describing a perfect fluid coupled with a poloidal magnetic field for the line element (2) is

$$
T^{\mu v}=\left(\begin{array}{cccc}
\frac{1}{A^{2}}(\rho+W) & 0 & 0 & 0 \\
0 & \frac{1}{B^{2}}(P-\Pi) & \frac{1}{r B^{2}} \sigma & 0 \\
0 & \frac{1}{r B^{2}} \sigma & \frac{1}{(B r)^{2}}(P+\Pi) & 0 \\
0 & 0 & 0 & \frac{1}{D^{2}}(P+W)
\end{array}\right) .
$$

The first term, i.e., $T^{00}$ in Equation (24) represents the total energy density of the system which comes from the perfect fluid distribution and the electromagnetic field, through the quantity $W$; the others diagonal terms correspond to the pressure and as we can see the quantities $\Pi$ and $W$, which depend on the electromagnetic four potential, and compose the total pressure of the system. Finally, 
the off-diagonal terms depend only on the electromagnetic four potential and represents the shear stress of the system $\sigma$.

With the goal of providing a physical interpretation to the quantities $W, \Pi$ and $\sigma$ we now derive the conservation equations for a perfect fluid coupled with a poloidal magnetic field and compare these equations with those obtained in [39] where no electromagnetic contribution was considered.

The non-vanishing components of the conservation equations $T_{; v}^{\mu v}=0$ for the energy-momentum tensor (24) are

For $\mu=0$

$$
\dot{\rho}+\dot{W}=0
$$

where the dot denotes derivative with respect to $t$. Equation (25) is a consequence of the staticity.

The other non-vanishing components are

$\mu=1$

$$
\begin{aligned}
(P-\Pi)^{\prime}+\frac{A^{\prime}}{A}(\rho+W+P-\Pi) & -\frac{B^{\prime}}{B} 2 \Pi-\frac{D^{\prime}}{D}(W+\Pi)+ \\
& +\frac{1}{r}\left[\sigma_{, \theta}+\left(\frac{A_{, \theta}}{A}+2 \frac{B_{, \theta}}{B}+\frac{D_{, \theta}}{D}\right) \sigma-2 \Pi\right]=0
\end{aligned}
$$

$\mu=2$

$$
\begin{aligned}
(P+\Pi)_{, \theta}+\frac{A, \theta}{A}(\rho+W+P+\Pi) & +\frac{B_{, \theta}}{B} 2 \Pi-\frac{D_{, \theta}}{D}(W-\Pi)+ \\
& +r\left[\sigma^{\prime}+\left(\frac{A^{\prime}}{A}+2 \frac{B^{\prime}}{B}+\frac{D^{\prime}}{D}\right) \sigma\right]+2 \sigma=0,
\end{aligned}
$$

where $f^{\prime}$ and $f_{, \theta}$ means derivative with respect the coordinates $r$ and $\theta$, respectively. Equations (26) and (27) are the hydrostatic equilibrium equations. In the special case of vanishing magnetic field and istropic fluid, these equations correspond to the Tolman-Oppenheimer-Volkoff Equations [40-42].

Herrera et al. [39], studied axially symmetric, static bound sources. The matter content considered for the authors in a local Minkowski coordinates $(\tau, x, y, z)$ is given by

$$
\hat{T}_{\alpha \beta}=\left(\begin{array}{cccc}
\mu & 0 & 0 & 0 \\
0 & P_{x x} & P_{x y} & 0 \\
0 & P_{y x} & P_{y y} & 0 \\
0 & 0 & 0 & P_{z z}
\end{array}\right),
$$

where $\mu, P_{x x}, P_{y y}, P_{z z}, P_{x y}=P_{y x}$ denote the energy density and different stresses, respectively, measured by a local Minkowski observer. In a spacetime described by (2), the canonical form of the energy-momentum tensor is

$$
T_{\alpha \beta}=(\mu+P) V_{\alpha} V_{\beta}+P g_{\alpha \beta}+\Pi_{\alpha \beta},
$$

with

$$
\begin{aligned}
\Pi_{\alpha \beta} & =\left(P_{x x}-P_{z z}\right)\left(K_{\alpha} K_{\beta}-\frac{h_{\alpha \beta}}{3}\right) \\
& +\left(P_{y y}-P_{z z}\right)\left(L_{\alpha} L_{\beta}-\frac{h_{\alpha \beta}}{3}\right)+2 P_{x y} K_{(\alpha} L_{\beta)}, \\
P & =\frac{P_{x x}+P_{y y}+P_{z z}}{3}, \quad h_{\alpha \beta}=g_{\alpha \beta}+V_{\alpha} V_{\beta},
\end{aligned}
$$


where

$$
V_{\alpha}=(-A, 0,0,0), \quad K_{\alpha}=(0, B, 0,0), \quad L_{\alpha}=(0,0, B r, 0)
$$

The conservation equations calculated by [39] are

$$
\begin{aligned}
P_{x x}^{\prime}+\frac{A^{\prime}}{A}\left(\mu+P_{x x}\right)+\frac{B^{\prime}}{B}\left(P_{x x}-P_{y y}\right)+ & \frac{D^{\prime}}{D}\left(P_{x x}-P_{z z}\right)+ \\
\frac{1}{r}\left[P_{x y, \theta}+\left(\frac{A_{, \theta}}{A}+2 \frac{B_{\theta},}{B}+\frac{D_{, \theta}}{D}\right) P_{x y}+P_{x x}-P_{y y}\right]=0, & \\
P_{y y, \theta}+\frac{A_{, \theta}}{A}\left(\mu+P_{y y}\right)+\frac{B_{, \theta}}{B}\left(P_{y y}-P_{x x}\right)+ & \frac{D_{, \theta}}{D}\left(P_{y y}-P_{z z}\right)+ \\
& \quad \begin{array}{r}
{[} \\
{\left[P_{x y}^{\prime}+\left(\frac{A^{\prime}}{A}+2 \frac{B^{\prime}}{B}+\frac{D^{\prime}}{D}\right) P_{x y}\right]+2 P_{x y}=0 .}
\end{array}
\end{aligned}
$$

Comparing Equations (26) and (27), which describe a perfect fluid coupled with a poloidal magnetic field, with the hydrostatic Equations (33) and (34), calculated in [39], which describe an anisotropic fluid (without electromagnetic contribution), we can read the quantities $\rho+W$ as the total energy density of our distribution. In fact, the definition of $W$ given by Equation (20) remind us of the typical definition of the electromagnetic energy density. The quantity $2 \Pi$ can be read as the anisotropy of the distribution, and it is a direct consequence of the poloidal magnetic field. The quantity $\sigma$ given by (22) can be identified as the shear stress experienced by the fluid. The quantities $W+\Pi$ and $W-\Pi$ can be read as an anisotropy defined with respect to z-axis. In conclusion, if we apply the Bondi approach [43] then a locally Minkowskian observer measures, for the perfect fluid coupled with a poloidal magnetic field, $\rho+W$ as the total energy density, $2 \Pi$ as the anisotropy caused by the different components of the magnetic field and $\sigma$ as the shear stress experienced by the fluid.

\subsection{Global Structural Properties}

In the previous section we have addressed the formal aspects of the magnetic field in a general relativistic, axis-symmetric environment. Such studies are useful in aiding us in understanding how the presence of a poloidal magnetic field alters the geometry of star and to properly identify relevant quantities such as anisotropy, shear stress and energy density. One must, however, resort to numerical calculations if one wants to quantitatively describe such alterations in the structure of neutron stars. With that in mind we now move forward to discuss numerical results of the effects of magnetic fields on macroscopic stellar properties, obtained from using the LORENE (Langage Objet pour la RElativité NumériquE) code $[37,44]$. In this axisymmetric formalism, a global poloidal magnetic field is generated though a global current and the field strength generated depends on the stellar radius, angle $\theta$ (with respect to symmetry axis), and dipole magnetic moment $\mu$, being different for each equation of state. Note that different approaches agree that the maximum central magnetic field inside neutron stars cannot be larger than a couple of times $10^{18} \mathrm{G}[37,44-47]$.

As numerical calculations show, there are substantial changes in masses and radii for neutron stars that possess strong magnetic fields, but the change comes mainly form the pure magnetic field contribution to the energy momentum tensor, which is highly anisotropic, as discussed in the previous section. Figure 2 shows the gravitational stellar mass and central enthalpy for families calculated with a fixed dipole magnetic moment. The different lines for each color show (1) effects only from the pure magnetic field contribution, (2) effects from pressure anisotropy and pure the magnetic field contribution, and (3) the effects from Landau levels, pressure anisotropy, and the pure magnetic field contribution. Note that fixed baryon masses (in the case of isolated stars) would imply moving up but at the same time left in the figure, in a way that central enthalpy decreases with magnetic field strength. For more details on the value for the mass increase in magnetic stars (which is of the order of percents in the case of fixed baryonic mass), see for example [27,48]. Note that it has been shown that such decrease in central enthalpy/chemical potential/density is equation of state and stiffness dependent [48]. 




Figure 2. Equilibrium sequence obtained from the numerical solution of he Einstein-Maxwell equations for the CMF model equation of state shown for different dipole magnetic moments including different magnetic field effects.

In this formalism, the stellar radii are largely modified, as poloidal magnetic fields generate oblate stars, with increased/decreased values of about tens or percent in the equatorial/polar direction, respectively. For extreme magnetic fields, for instance, the ratio $r_{\text {polar }} / r_{\text {equatorial }}$ can reach values near to 0.5 in the more extreme configurations (near the critical magnetic field after which the star starts to attain a torus geometry). One must note that such extreme configuration and the critical values of the magnetic field depend on the equation of state properties.

Note however that different stellar shapes are generated when toroidal magnetic fields are considered [49]. Although it is understood that the long-term stability of magnetic stars requires poloidal and toroidal components, those configurations are much more expansive to model numerically; see Ref. [30].

\section{Evolution Aspects}

Finally, we now investigate evolutionary aspects of the magnetic field. One must notice that in previous sections the aspects of the magnetic fields on neutron stars were studied under the assumption that such fields are static (or quasi-static). This does not need to be the case, and, in fact, observations show that the magnetic field in neutron stars is evolving. In this section we propose a phenomenological model for the evolution of magnetic fields that may affect indirectly (by masking the true age of the star) and directly (by generating heat) the thermal evolution of magnetized neutron star. We note, however, that we perform such studies independently, that is, ignoring micro and macroscopic effects. We stress that a complete, self-consistent study must take all of this into account, which is our ultimate goal for future investigations. For now, we focus in the objects known as XDINS. These are a few objects first detected by the ROSAT All-Sky Survey that are radio quiet but exhibit bright emission in the soft X-Ray band [50]. These objects do not exhibit non-thermal hard emission and their spectra are blackbody-like [51]. Furthermore, there is no known association with any supernova remnant, which could indicate that these objects cannot be too young [51]. Considerable information regarding the spin properties of XDINS has been collected along the years; see Table 1 for a summary of them. 
Table 1. Observational data for Isolated X-ray Neutron Stars (XDINS).

\begin{tabular}{cccccc}
\hline XDIN & $\begin{array}{c}\text { Period (s) } \\
\text { Name }\end{array}$ & $\begin{array}{c}\text { Period Derivative } \\
\dot{\boldsymbol{P}}\left(\mathbf{s} \mathbf{~ s}^{-\mathbf{1}} \mathbf{)}\right.\end{array}$ & $\begin{array}{c}\text { Blackbody Temperature } \\
\boldsymbol{T}_{\boldsymbol{b} \boldsymbol{b}} \mathbf{( K )}\end{array}$ & $\begin{array}{c}\text { References } \\
\text { R }\end{array}$ & $\begin{array}{c}\text { Spin-Down Age (Years) } \\
\boldsymbol{t}(\mathbf{y r})\end{array}$ \\
\hline RX J0720.4-3125 & 8.39 & $6.98 \times 10^{-14}$ & $9.7 \times 10^{5}$ & {$[52,53]$} & $1.9 \times 10^{6}$ \\
RX J0806.4-4123 & 11.37 & $5.5 \times 10^{-14}$ & $1.17 \times 10^{6}$ & {$[53,54]$} & $3.3 \times 10^{6}$ \\
RX J1308.6+2127 & 10.31 & $1.12 \times 10^{-13}$ & $1.09 \times 10^{6}$ & {$[53,55]$} & $1.5 \times 10^{6}$ \\
RX J1856.5-3754 & 7.055 & $2.97 \times 10^{-14}$ & $7.30 \times 10^{5}$ & {$[56,57]$} & $3.8 \times 10^{6}$ \\
RX J2143.0+0654 & 9.437 & $4.14 \times 10^{-14}$ & $1.24 \times 10^{6}$ & {$[58,59]$} & $3.6 \times 10^{6}$ \\
RX J0420.0-5022 & 3.45 & $2.8 \times 10^{-14}$ & $6.0 \times 10^{5}$ & {$[58,59]$} & $2.0 \times 10^{6}$ \\
RX J1605.3+3249 & 3.39 & $1.6 \times 10^{-12}$ & $1.15 \times 10^{6}$ & {$[60,61]$} & $3.4 \times 10^{4}$ \\
\hline
\end{tabular}

As shown in Table 1, the temperature obtained from their blackbody-like spectra (in the range of $0.6-1.4 \times 10^{6} \mathrm{~K}$ ) is normally associated with young neutron stars, however their long periods and absence of radio emission (both of which are normally observed in young neutron star) may indicate that these objects are not too young. Furthermore age estimations based on their spin-down properties, namely the spin-down age $\tau_{S D} \equiv P /(2 \dot{P})$, indicate very old ages. Evidently, the spin-down properties may not be a good indicator for the true age of the neutron stars [53], since it makes a series of strong assumptions, in particular with respect to the magnetic field that is assumed to be constant throughout the evolution. On the other hand, if the temperature of these objects is to be explained solely due to their young age, they would have to been born with very high magnetic fields or unusually long periods, as to explain how they can attain such long periods during their thermal evolution life [51]. This presents a challenge: how to explain such objects that show temperature of young neutron stars and rotation properties of old ones, when evidence indicates that they can be neither? We attempt to answer this question by computing the neutron star thermal evolution combined with a magnetic field evolution model. We show that the combination of their age, masked by the magnetic field evolution, plus the thermal emission, and in some cases possibly with some moderate internal heating source, could indicate the true age of these pulsars to lie in the intermediary range, i.e. between young and old ages. This result is in agreement with recent measurements of the proper motion of three XDINS, that indicate the age of at least of these stars approximate to be $4 \times 10^{5}$ years.

For that purpose we consider the thermal evolution of these objects, by making use of state of the art cooling calculations [62-64]. We employ the most recent thermal emission and cooling mechanisms available, that have also been used to explain the thermal properties of the cooling neutron star in Cassiopeia A [62,65-67]. In addition, we also consider a phenomenological model for the magnetic field evolution, first considering the magnetic field decay that could be used to explain the heat deposit in the neutron star crust [51], and also the magnetic field emergence (growth) after an initial hyper-critical accretion, followed by a final magnetic field decay (see Refs. [68-71], and references therein). We show how the thermal/spin properties of the XDINS may be explained in such scenario with and without the need of a heating mechanism.

\subsection{Magnetic-Thermal Evolution}

Here we aim to reconcile the observed properties of XDINS (in particular their thermal behavior) with the current trends on the theory of neutron star cooling. As mentioned before, if one uses the current models for neutron star thermal evolution and the observed properties of XDINS, namely $T_{b b}$ and the spin-down age $P /(2 \dot{P})$, one obtains a clear disagreement, as shown in Figure 3.

For the cooling calculation of Figure 3 we used a neutron star whose microscopic composition and equation of state (EoS) is given by the APR model [72]. The neutron star mass for this particular calculation is $1.55 M_{\odot}$. Furthermore neutron and proton pairing are assumed to take place in the star such that agreement with Cassiopeia A is possible [65-67]. Figure 3 shows that the XDINS seem to be much warmer than what is predicted for their ages. This would indicate that, either there is a powerful heating mechanism (unbeknownst to us) that is keeping these objects warm at old ages, or, these objects are much younger than their apparent age. There is, of course, the possibility of both. 


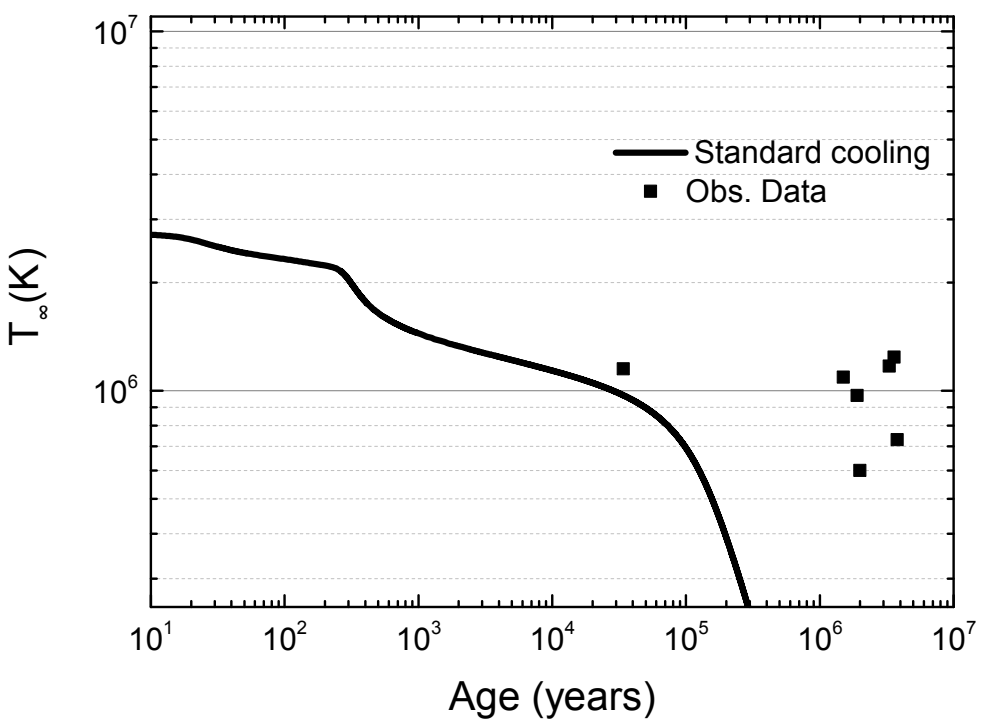

Figure 3. Temperature evolution of a $1.5 M_{\odot}$ neutron star. The squares represent the observed temperature of the XDINS at their respective spin-down age.

As discussed above, the spin-down age is a far cry from a reliable measurement of the true age of a neutron star. In the few cases in which both the spin-down age and the kinematic age have been estimated, they were usually off by thousands of years [73]. Even for XDINS, the few cases in which it was possible to estimate the kinematic age, it has been found that the neutron star must be younger than what their spin-down age indicates (see $[53,74]$, and references therein). A possible way in which the spin-down age of an object would be different than the actual age of the object is if the magnetic field of the star evolves over time. Several mechanisms have been proposed to explain the variation of the magnetic field, e.g., ohmic dissipation and ambipolar diffusion (both responsible for the decrease of the magnetic field) [53]. There has also been proposed that the magnetic field may be initially buried in the neutron star crust, due to a stage of hyper-critical accretion $[71,75,76]$, followed by an increase over time [70].

Here, rather than assuming a particular model for the magnetic field evolution, we choose a phenomenological approach, that gives us the freedom to investigate different relaxation and evolution times for the magnetic field, as to investigate under which conditions the magnetic field evolution may lead to an agreement with the observed spin-down age and thermal properties of XDINS. This study does not exclude the possibility of heating also taking place; actually, the agreement with observed data can only be improved if heating is present.

We start considering the possibility of the object being born with a magnetic field that undergoes a decay over time. We adopt a function for the magnetic field decay in the form of a smoothed top-hat with the parameter $\tau_{D}$ indicating the relaxation time. We study different relaxation times for the magnetic field decay, as indicated in Figure 4.

Applying the magnetic breaking model for such magnetic field evolution, one can find the relevant rotation quantities, such as the rotation period and period time derivatives, which allow us to calculate the estimated spin-down age, $\tau_{c}=P /(2 \dot{P})$, and contrast it to the real age of the object. This is shown in Figure 5 . We must stress that the spin-down age $\tau_{c}=P /(2 \dot{P})$ is only valid for the canonical case where the magnetic field is constant, which, evidently is not the case here. We have, however, chosen to use the quantity $\tau_{c}=P /(2 \dot{P})$ as a parameter, even though we are not considering a constant magnetic field, since this is how the spin-down age is estimated based on observational data (such as that in Table 1). Therefore, by doing that, we make sure we are comparing the same quantities. This is, in fact, an important point of this work, that we must look at the quantity $\tau_{c}=P /(2 \dot{P})$ as an observational parameter, rather than a true reflection of the age of the star. 




Figure 4. Different magnetic field evolution functions used to represent a neutron star undergoing magnetic field decay. $\tau_{\mathrm{D}}$ represents the magnetic field relaxation time.

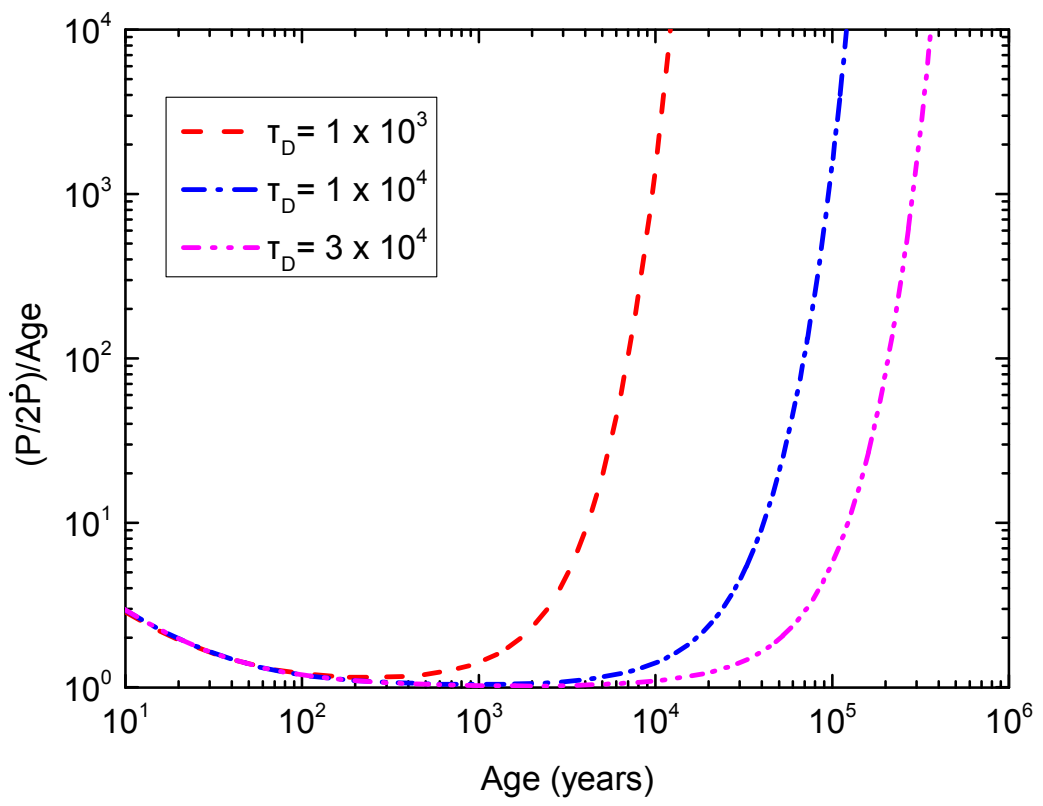

Figure 5. Estimated spin-down age $P /(2 \dot{P})$ over the true age of the pulsar as estimated for the magnetic field evolutions of Figure 4.

As one can see in Figure 5, in the initial stages of evolution, the spin-down age differs very little from the true age. At later stages of evolution, we have a deviation from the canonical case, with the spin-down age indicating an age much older than the actual age of the star. This result shows, as discussed before, that comparing the observed temperature of neutron stars with their observed spin-down age may erroneously indicate than an object retains high temperature at later ages. We show this by calculating the temperature evolution of the same neutron star used in Figure 3, undergoing the magnetic field evolution depicted in Figure 4. These results are shown in Figure 6. 


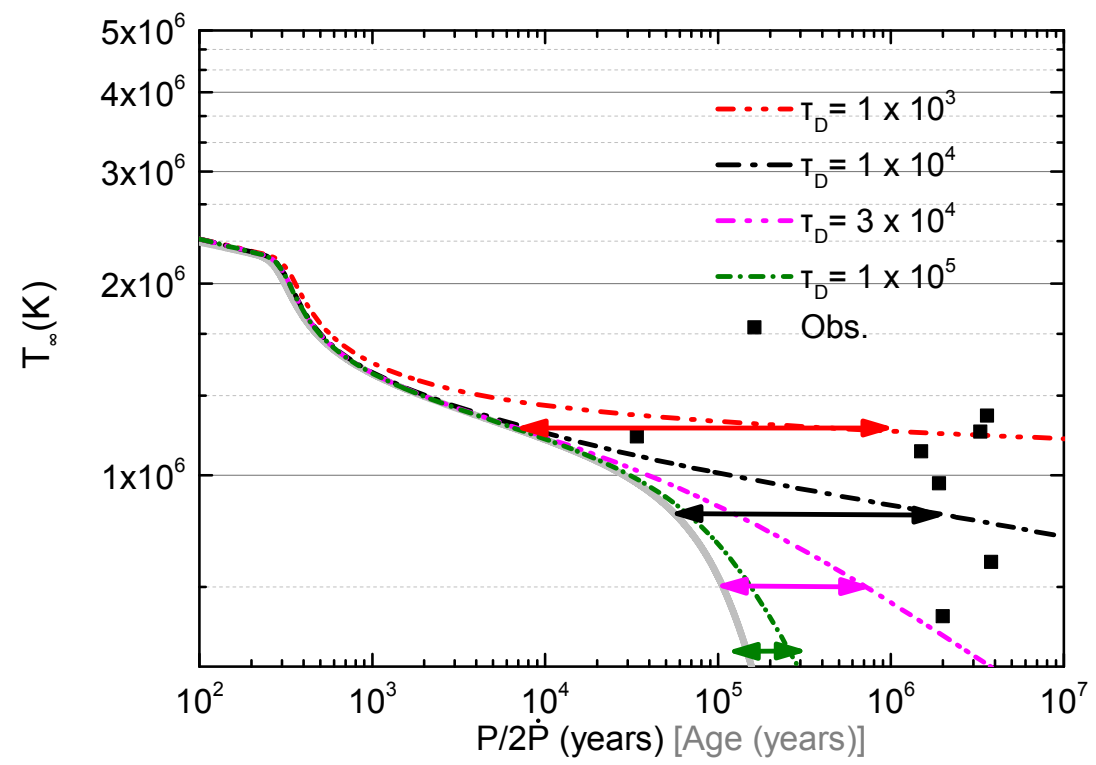

Figure 6. Thermal evolution of a $1.55 M_{\odot}$ neutron star undergoing magnetic field decay. The solid washed gray line represents the temperature as a function of the real age of the object. For the other curves the $x$-axis represent the spin-down age observed. We also plot a few arrows depicting the discrepancy between the true and spin-down age.

As indicated by Figure 6, by using the spin-down age as a measurement of the age of the object, one may believe that a neutron star is much older than reality. One also sees that for stronger magnetic field decay (those that take place more quickly, with lower relaxation times), the deviation from the real age of the object becomes more pronounced. For instance, as can be seen from Figure 6, a star with surface temperature of $10^{6} \mathrm{~K}$ may appear to be $10^{6}$ years old as measured by the spin-down age while, if we assume that the magnetic field is decaying with $\tau_{\mathrm{D}}=10^{3-4}$ years, the star has a real age of approximately $5 \times 10^{4}$ years (see red arrow in Figure 6). The interesting fact of this result is that, if the magnetic field is indeed undergoing a decay process, we may use the observed temperature to estimate the age of the star, or, conversely, if we know the age of the star from other measurements, for instance kinematic estimates, we can then determine how the magnetic field of the object is evolving.

Based on the results just discussed, we can conclude that if the only reason of these objects appearing to be warm at old ages is due to magnetic field decay, the warmer the temperature of the object the stronger the magnetic field decay needs to be. This is due to the fact that high temperatures are associated with younger stars (if no heating is assumed, as is the case for now), thus only with a strong magnetic field decay one can explain the high deviation from the real age of the object. For lower observed temperatures, a moderate magnetic field decay is enough, since a lower temperature does not require very young ages. Our conclusion is that for objects with the highest temperatures it is unlikely that only the magnetic field decay and the consequent deviation from real age are enough to explain their observed properties. For those cases, a heating source may be necessary. For the high temperature objects in Table 1 , if no heating is present, the real age of the star would be $\sim 10^{3}$ years, and the magnetic field would have to strongly decay in a matter of $10^{3}$ years as well. This seems to be an unlikely scenario. As for the objects with lower temperatures, one may not need to resort to heating, since a moderate magnetic field decay with relaxation times $\tau_{\mathrm{D}}=10^{3-5}$ years would be enough to explain their temperatures, and the real age would be around $10^{4-5}$ years. It is important to notice that measurements of the proper motion of three of the magnificent seven have yielded to kinematic ages lower than the spin-down ones [53]; Furthermore, as mentioned before, the new distance calculated for RXJ1856.5-3754 [74], implies a kinematic age of $5 \times 10^{4}$ years, in agreement with the age we estimate for the moderately warm XDINS. 


\subsection{Complex Magnetic Field Evolution}

In addition to the above study of the magnetic field decay scenario, we proceed now to consider as well a somewhat more complex situation. As it has been proposed in Refs. [68,71,77], after a hyper-accretion phase at the early stages of evolution, the magnetic field of a neutron star may be buried into the crust of the object. In the stellar core-collapse scenario (including catastrophic collapse of an iron core, generating a shock wave, and the dramatic ejection of the star envelope), the residual compact remnant could be a neutron star immersed in a dense environment. Such just born neutron star may be exposed to an hyper-accretion phase from fallback material a few seconds after the supernova event [78]. When the ejected matter collides with denser external layers, it is bifurcated and a reverse shock is formed. In that case, some part of ejected material continues on its way to the outside where the hydrodynamics effects are not very important, while another part of the material fallback onto the surface of the proto-neutron star. This reverse shock allows to deposit large amount of matter onto the proto-neutron star surface forming a new crust and hiding there any initial configuration of the magnetic field of the star, as it was shown in Ref. [71]. In that case, the magnetic field may be buried. After the hyper-accretion phase ends and the residual envelope of the newborn neutron star vanishes, the magnetic field can suffer a growth phase, emerging from the crust and appearing as a delayed-off pulsar, as it was pointed out in $[68,70]$. When the magnetic field is buried on the stellar surface, it can be amplified by compression due to the strong accretion and by a turbulent dynamo. In this scenario, the magnetic field could grow to higher values than those estimated for XDINS and then suffer the familiar decay stage as we studied above.

We now investigate such scenario by considering, as before, a phenomenological evolution for the magnetic field that takes into account the emergence and decay phase. We consider a two smoothed top-hat analytic function that take into account the early growth and the later decay. We consider all growth to have the same relaxation time, as indicated in Figure 7, and only vary the relaxation time of the magnetic field decay $\left(\tau_{\mathrm{D}}\right)$. Such magnetic field evolution is represented in Figure 7.

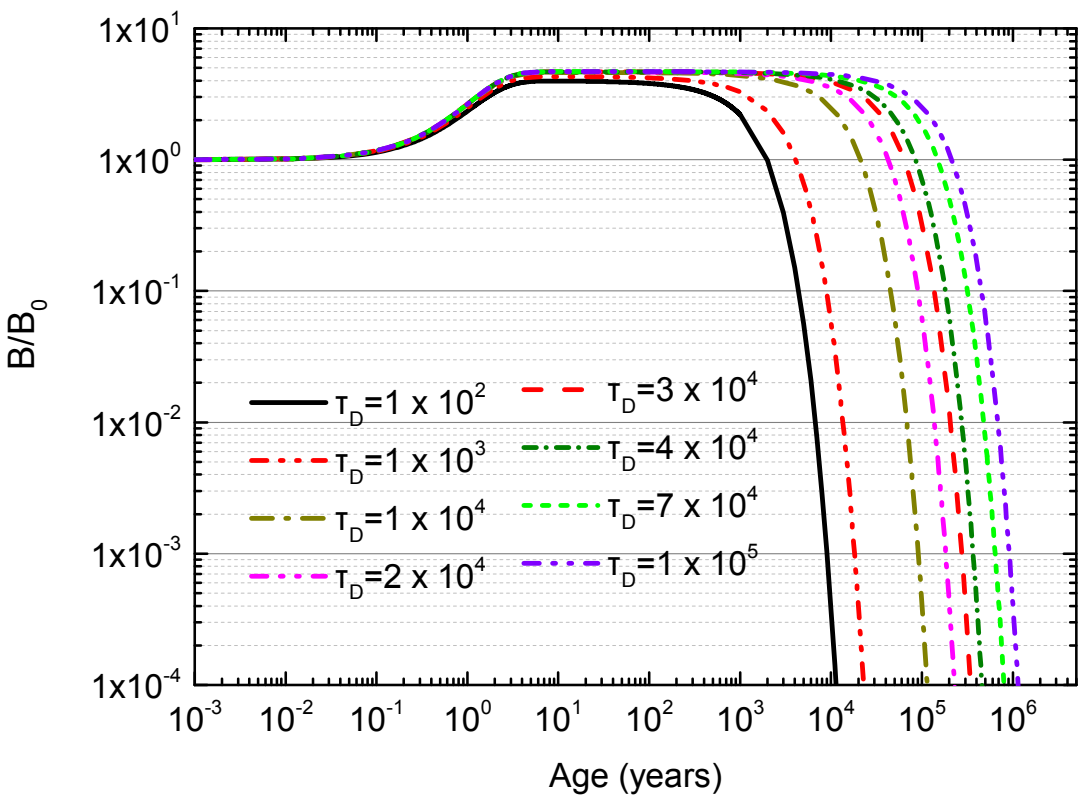

Figure 7. Magnetic field evolution representing the emerging after a hyper-accretion phase followed by the decay of the field. $\tau_{D}$ represents the relaxation time of the decay phase.

We show in Figure 8 the spin-down age over the true age of the star of a neutron star whose magnetic field undergoes such an evolution. This result shows us that initially the spin-down age is slightly higher than the true age (reflecting the growth of the magnetic field), followed by the a 
behavior equal to the canonical case, since once again the magnetic field is constant. Later, at the onset of the magnetic field decay, we see the same deviation from the canonical case as the previously studied case, with the spin-down age indicating objects older than their real age. As expected, since at this epoch the behavior is the same as in the decay-only scenario, we see that for stronger (faster) magnetic decays we obtain stronger deviations from the canonical case.

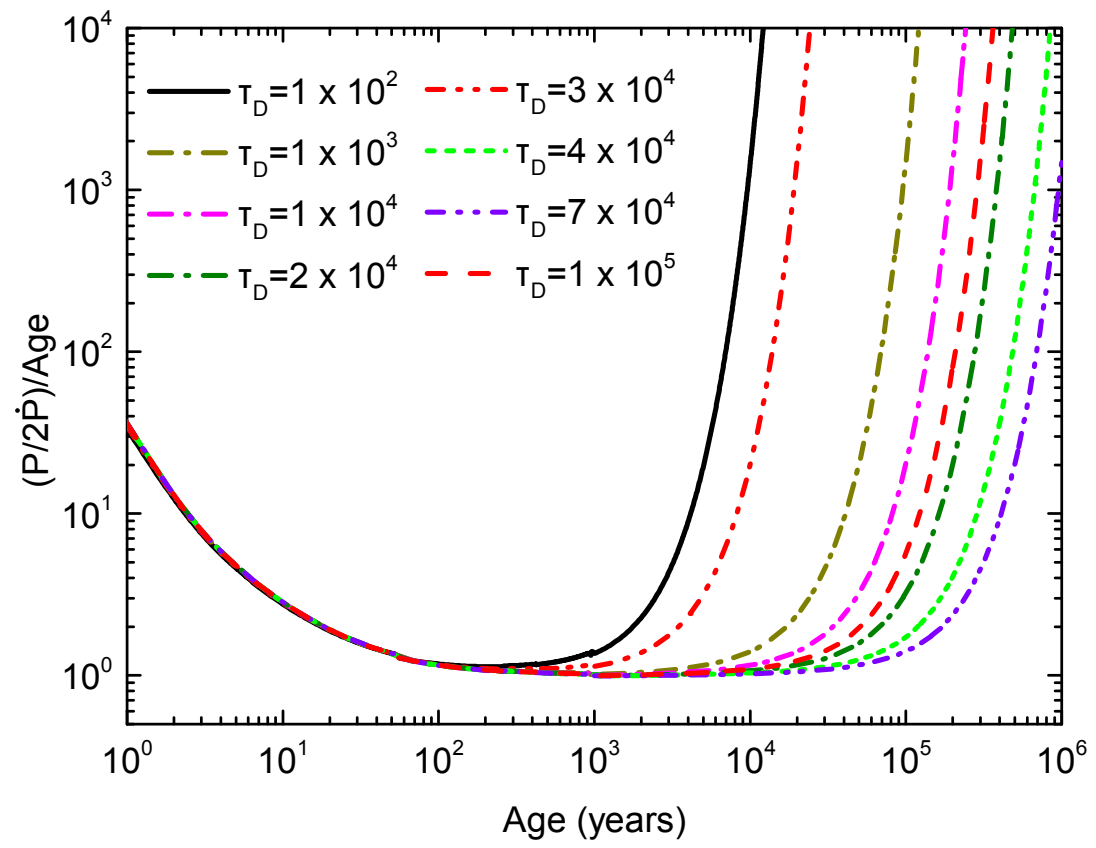

Figure 8. Spin-down age $(P / 2 \dot{P})$ over the true age of the star as a function of the real age for a neutron star whose magnetic field emerges, after the burial due to a hyper-accretion phase, and then it is followed by a decay.

We now calculate the thermal evolution of the same neutron stars that we have followed in this investigation, except now considering such magnetic field evolution. This, as it was the case before, allows us to keep track of their real and spin-down age and, by using the observed temperature and spin-down parameters (particularly $P$ and $\dot{P}$ ), we can probe which magnetic field evolution would be appropriate to explain such observed quantities without resorting to any heating mechanisms. The thermal evolution calculations are shown in Figure 9.

As the case we studied before, we see that the magnetic field decay may lead one to believe that a neutron star is much older than reality (see black arrow in Figure 9). The emergence of the magnetic field has little impact on the spin-down age at later stages, thus we cannot use the thermal evolution to determine whether or not such emergence occurs. The same conclusions for the decay-only case hold here: the best scenario to describe the observed properties of the XDINS (without resorting to any heating mechanism) would be a magnetic field decay with relaxation time of the order of $\tau_{\mathrm{D}}=10^{3-5}$ years. This is true to the objects that are moderately warm, those that are hotter would need a more violent magnetic field decay, which would also indicate that they are actually much younger. As mentioned before, we believe that this is an unlikely scenario, due to the lack of evidence that such objects are much younger than $10^{4}$ years. 


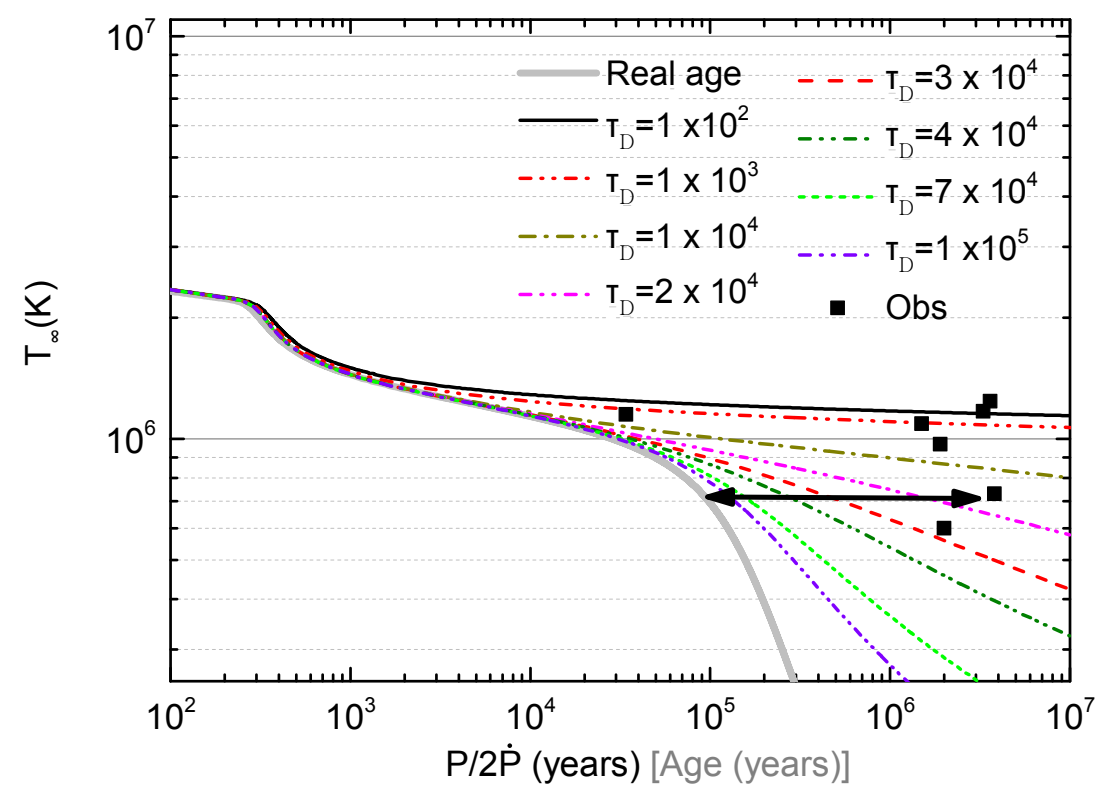

Figure 9. Thermal evolution of neutron stars whose magnetic field evolve according to Figure 7. With the exception of the washed gray curve, which shows the real age, the curves represent the temperature as observed by an observer at infinity as a function of the spin-down age $(P / 2 \dot{P})$. We also indicate by the arrow an example of the discrepancy between the true and spin-age (to avoid cluttering the image we only show one arrow).

Finally, we briefly consider the presence of heating, as to demonstrate that our model does not invalidate such scenario and that, in fact, it benefits from it. As mentioned before, we believe that the warmer objects of Table 1 would be better explained by a combination of heating and magnetic field decay. We have seen that the latter alone would indicate that these objects are undergoing a rather fast magnetic field decay (of the order of 100-1000 years) and that they would need to be very young (as to explain their high temperature). On the other hand, if their temperature is to be explained solely by heating, a considerable amount of continuous heating would be needed to keep such objects so warm at such old ages. We thus consider a heating source that is uniformly distributed along the crust of the neutron star, with an intensity of $H \approx 10^{16} \mathrm{erg} \mathrm{s}^{-1} \mathrm{~cm}^{-3}$, that is active for $\sim 10^{4}$ years. The magnetic field evolution is the same as we have used in the previous calculations.

As it is shown in Figure 10, the presence of a heating increases the temperature of the neutron star while the heating source is active. With this, one can now explain the high temperature observed in a few XDINS as a result of a magnetic field decay with $\tau_{D} \sim 10^{4}$ years combined with a heating acting with a relaxation time also of $\sim 10^{4}$ years. In this scenario, as indicated in Figure 10, the true age of the object would be $\sim 10^{5}$ years, rather than $\sim 10^{3-4}$ years, as it would be in the absence of heating. Evidently, one could argue that a hypothetical source of heat could be active for $10^{6-7}$ years, thus solely explaining the high temperature of these stars. We cannot rule out this possibility; however, we believe that the combination of moderate heating plus a magnetic field evolution that masks the true age of the star is a more realistic scenario, since the explanation based only on the existence of a heating source would need a very large internal energy-power $\left(\dot{E}_{H} \sim 10^{34} \mathrm{erg} \mathrm{s}^{-1}\right)$ active for very long times of the order of $10^{6-7}$ years. 


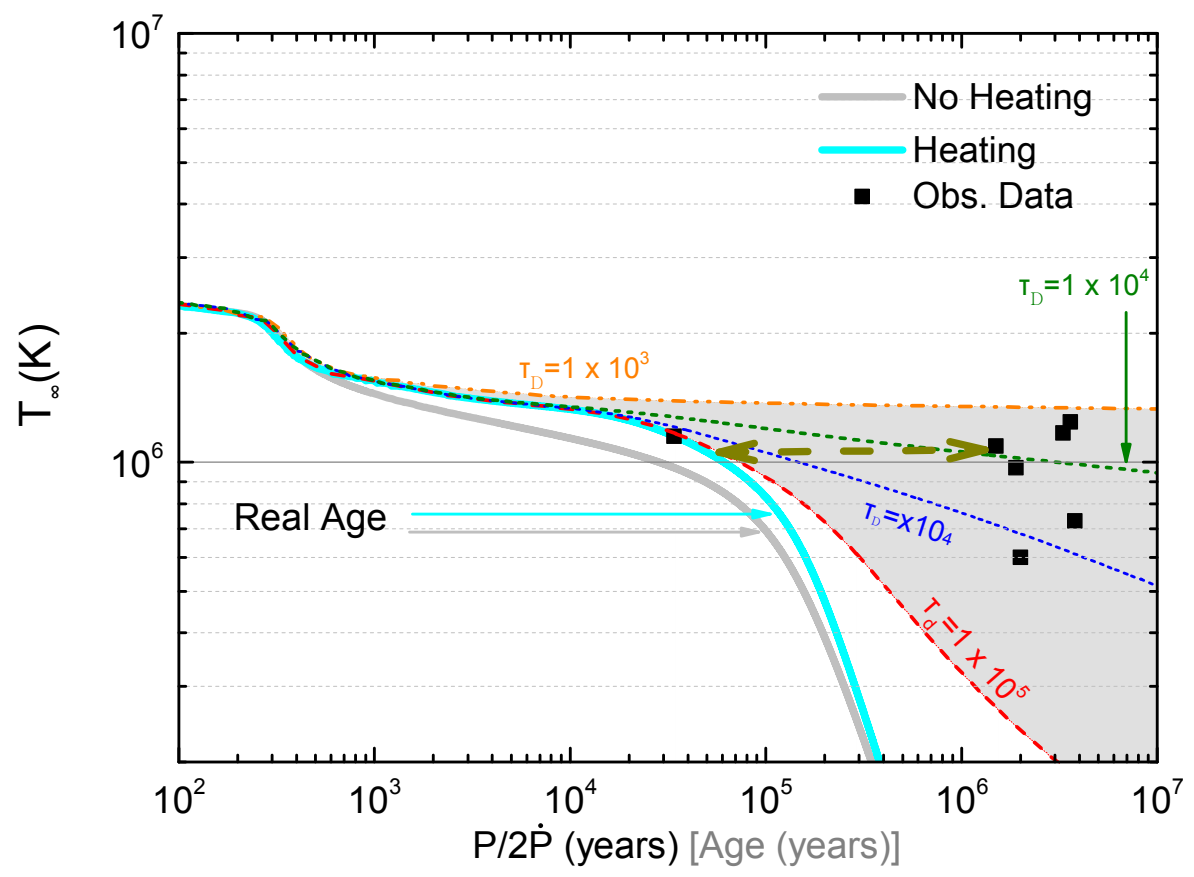

Figure 10. Same as Figure 9 but with a heating source of $H \approx 10^{16} \mathrm{erg} \mathrm{s}^{-1} \mathrm{~cm}^{-3}$ uniformly applied to the crust for the first $10^{4}$ years. Curves accompanied by the indicated $\tau_{\mathrm{D}}$ represent the temperature as a function of the spin-down age $(P / 2 \dot{P})$. The shaded area represents where the curves for magnetic field evolutions with $\tau_{\mathrm{D}}=10^{3-5}$ years lie. We also indicate, for the sake of example, the difference between the spin-down age and real age (in the presence of heating) for the XDINNS RXJ130862127.

Our results indicate that the magnetic field decay works well to explain the moderately warm XDINS (with $T<10^{6} \mathrm{~K}$ ). For the higher temperature XDINS, such scenario seems far fetched since it would require a rather fast magnetic field decay $\left(\tau_{\mathrm{D}} \sim 10^{2-3}\right.$ years) and a stellar true age of $10^{3-4}$ years. We believe that for such objects a moderate source of heating might be necessary. To illustrate that, we considered a heating source of $H \approx 10^{16} \mathrm{erg} \mathrm{s}^{-1} \mathrm{~cm}^{-3}$ acting in approximately the same timescale of the magnetic field decay, i.e., $10^{4}$ years. We find that with such heating, the magnetic field decay shows a more palatable situation for the warmer XDINS, that may, under this scenario, be explained with a magnetic field decay with $\tau_{\mathrm{D}}=10^{4}$ years, leading to a true age of $10^{4-5}$ years. We stress, once again, that we cannot rule out the presence of intense heating that acts on the neutron star for $10^{6-7}$ years, making the magnetic field decay unnecessary. We believe, however, that the combination of magnetic field decay with a moderate heating source is a more realistic scenario, specially in view of the recent results regarding the kinematic age of a few XDINS [53,74] that indicate that such objects may be younger than the spin-down age indicates, with a true age $\sim 4 \times 10^{5}$ years.

One must note that other authors have investigated the evolution of magnetic fields taking a less phenomenological approach, based on numerical simulations; see, for instance, [79,80]. Such approach is certainly interesting, although different of our purpose in the research we present here, where rather than investigate a particular evolution model we chose to investigate a whole set of them, which, when compared to observed data, allow us to best choose the most likely behavior of the magnetic field evolution. Evidently we have no microscopic information, and we must eventually look for a microscopic model that agrees with the conclusions reached by our investigation, and for that one must resort to simulations as those found in $[79,80]$. Although beyond of the scope of this work, we have briefly compared our results with those of $[79,80]$, and found that they are, for the most part compatible. In these studies, the authors presented numerical results of the magneto-thermal evolution of isolated neutron stars, including the most relevant physical parameters. They found that certain thermodynamic mechanisms in the inner crust of the neutron star are responsible for the high 
dissipation of the magnetic field on time-scales $100 \mathrm{kyr}$, which is compatible with our phenomenological approach that allow us to explore interesting characteristics of such evolution without the need for intensive numerical work. We also note that the authors in [81] have shown that including Hall effect in their numerical simulations leads to a saturation of the magnetic field evolution within $100 \mathrm{kyr}$, allowing a slow and sustained decay. The conclusion is that the most energetic behavior occurs while the star is young (high magnetic fields involved). This issue support our results that a phase of growth of the magnetic field was subsequent to the hypercritical regime of the newborn neutron star.

Finally we stress that most data suggests an unified model for neutron stars whose key parameter is the magnetic field $[82,83]$. However, the magneto-thermal model does not explain how neutron stars may be born with such high magnetic fields. If the hidden magnetic field scenario is correct, there exists the possibility that the magnetic field can be amplified (by two or three orders of magnitude) due a turbulent dynamo effects inside the neutron star crust in a timescale of months, and then subsequently undergo a phase of growth appearing as a delayed switch-on pulsar, several years after the supernova event, which may explain the highest magnetic fields associated with magnetars. Following that, the neutron star continues its canonical evolution until the thermo-magnetic effects can be relevant and cause the magnetic field to decay at the aforementioned time-scales. This phase of amplification of the magnetic field in the crust requires an intensive numerical treatment that is outside the scope of this work. But we aim to explore this possibility in a future work, as well as coupling such evolution to microscopic and macroscopic studies.

\section{Conclusions}

In this work we studied many ways in which a magnetic field may affect neutron stars, starting with the microscopic composition, going through the stellar structure, and culminating with evolutionary aspects. As we have shown, the proper account of the magnetic field in these different realms is no ordinary task. It is, however, imperative to do so. As we have seen, the magnetic field plays an important role in all regimes of the neutron star, altering its composition, affecting its structure and geometry, and being a key factor for the neutron star evolution.

For the composition, our study shows that the magnetic field may suppress the appearance of hyperons and the quark-matter phase transition, leading to a substantially different particle composition when compared to a neutron stars with small or vanishing magnetic fields.

In the macroscopic realm, we have discussed the formal aspects under which a dipolar magnetic field needs to be considered under the light of general relativity. We have shown that the presence of a dipolar magnetic field causes the fluid to become anisotropic and we identified the shear stress caused by it. We have also numerically calculated the structure of highly magnetized neutron stars and have shown that high magnetic fields can lead to more massive objects.

Finally we have shown that a better comprehension of the magnetic field evolution in neutron stars is fundamental for being able to accurately estimate the ages of XDINS. We propose to use XDINS as a way of probing the magnetic field evolution, by making use of the observed spin-down properties (particularly the spin-down age) and thermal properties. By making use of kinematic observations capable of making better estimates of the star's true age, we can use the temperature to determine the most likely scenario for the magnetic field evolution and/or presence of heating. We have found that for moderately warm XDINS there is no need of heating, and a magnetic field evolution with relaxation times of $\tau_{\mathrm{D}} \sim 10^{4-5}$ years is enough to explain their thermal properties, making their true age $\sim 10^{5}$ years. As for the warmer XDINS, we have found that a moderate heating is necessary, in addition to magnetic field decay with $\tau_{\mathrm{D}} \sim 10^{4}$ years, leading to a true age for these objects $\sim 10^{4-5}$ years.

As thoroughly discussed above we recall that as much as we strived to describe the different ways in which a magnetic field may affect neutron stars, we have certainly not covered all the possibilities. Furthermore, all of the studies we presented were done independently of each other, whereas a more appropriate (and challenging) treatment would consider all aspects on the same framework. That is our 
goal for future investigations. Considering that now we have a more comprehensive understanding of how the magnetic field affects these three realms (microscopic, macroscopic and evolutionary) we hope to perform self-consistent studies of all of them. That means that the equation of state (microscopic) would be affected by the macroscopic distribution of the magnetic field in the star, as well as the evolution of such fields. Evidently the macroscopic structure as well as the evolution of the object also depend on the microscopic equation of state, making such treatment highly non-linear and challenging, although still warranted if one aims to obtain a more complete description of neutron stars.

Acknowledgments: V.D. acknowledges support from NewCompStar COST Action MP1304. R.N. and O.T. acknowledges financial support from CAPES and CNPq. R.N acknowledges that this work is a part of the project INCT-FNA Proc. No. 464898/2014-5.

Author Contributions: All authors contributed equally to the formulation of the model, numerical analysis and writing of the paper.

Conflicts of Interest: The authors declare no conflict of interest.

\section{Reference}

1. Duncan, R.C.; Thompson, C. Formation of very strongly magnetized neutron stars-Implications for gamma-ray bursts. Astrophys. J. 1992, 392, L9-L13.

2. Thompson, C.; Duncan, R.C. Neutron star dynamos and the origins of pulsar magnetism. Astrophys. J. 1993, 408, 194-217.

3. Olausen, S.A.; Kaspi, V.M. The McGill Magnetar Catalog. Astrophys. J. Suppl. 2014, 212, 6.

4. Thompson, C.; Duncan, R.C. The soft gamma repeaters as very strongly magnetized neutron stars-I. Radiative mechanism for outbursts. Mon. Not. R. Astron. Soc. 1995, 275, 255-300.

5. Thompson, C.; Duncan, R.C. The Soft Gamma Repeaters as Very Strongly Magnetized Neutron Stars. II. Quiescent Neutrino, X-ray, and Alfven Wave Emission. Astrophys. J. 1996, 473, 322.

6. Kaspi, V.M.; Kramer, M. Radio Pulsars: The Neutron Star Population Fundamental Physics. ArXiv 2016, arXiv:astro-ph.HE/1602.07738.

7. Melatos, A. Bumpy spindown of anomalous X-ray pulsars: The link with magnetars. Astrophys. J. 1999, 519, L77.

8. Makishima, K.; Enoto, T.; Hiraga, J.S.; Nakano, T.; Nakazawa, K.; Sakurai, S.; Sasano, M.; Murakami, H. Possible Evidence for Free Precession of a Strongly Magnetized Neutron Star in the Magnetar 4U 0142+61. Phys. Rev. Lett. 2014, 112, 171102.

9. Landau, L.D.; Lifshitz, E.M. Quantum Mechanics: Non-Relativistic Theory; Elsevier: Amsterdam, The Netherlands, 1977.

10. Lai, D.; Shapiro, S.L. Cold equation of state in a strong magnetic field-Effects of inverse beta-decay. Astrophys. J. 1991, 383, 745-751.

11. Chakrabarty, S. Quark matter in strong magnetic field. Phys. Rev. D 1996, 54, 1306-1316.

12. Chakrabarty, S.; Bandyopadhyay, D.; Pal, S. Dense nuclear matter in a strong magnetic field. Phys. Rev. Lett. 1997, 78, 2898-2901.

13. Yuan, Y.F.; Zhang, J.L. The Effects of Interior Magnetic Fields on the Properties of Neutron Stars in the Relativistic Mean-Field Theory. Astrophys. J. 1999, 525, 950-958.

14. Broderick, A.; Prakash, M.; Lattimer, J.M. The Equation of state of neutron star matter in strong magnetic fields. Astrophys. J. 2000, 537, 351.

15. Dexheimer, V.; Negreiros, R.; Schramm, S. Hybrid stars in a strong magnetic field. Eur. Phys. J. A 2012, $48,189$.

16. Canuto, V.; Chiu, H.Y. Quantum theory of an electron gas in intense magnetic fields. Phys. Rev. 1968, $173,1210-1219$.

17. Canuto, V.; Chiu, H.Y. Thermodynamic properties of a magnetized fermi gas. Phys. Rev. 1968, 173, 1220-1228.

18. Strickland, M.; Dexheimer, V.; Menezes, D.P. Bulk Properties of a Fermi Gas in a Magnetic Field. Phys. Rev. D 2012, 86, 125032 .

19. Dexheimer, V.; Menezes, D.P.; Strickland, M. The influence of strong magnetic fields on proto-quark stars. J. Phys. G Nucl. Part. Phys. 2014, 41, 015203. 
20. Andersen, J.O.; Naylor, W.R.; Tranberg, A. Phase diagram of QCD in a magnetic field. Rev. Mod. Phys. 2016, 88,025001 .

21. Broderick, A.E.; Prakash, M.; Lattimer, J.M. Effects of strong magnetic fields in strange baryonic matter. Phys. Lett. B 2002, 531, 167-174.

22. Bordbar, G.H.; Rezaei, Z. Magnetized hot neutron matter: Lowest order constrained variational calculations. Phys. Lett. B 2013, 718, 1125-1131.

23. Dexheimer, V.; Negreiros, R.; Schramm, S.; Hempel, M. Deconfinement to Quark Matter in Neutron Stars-The Influence of Strong Magnetic Fields. AIP Conf. Proc. 2013, 1520, 264-269.

24. Weinberg, S. Why do quarks behave like bare Dirac particles? Phys. Rev. Lett. 1990, 65, 1181-1183.

25. Ferrer, E.J.; de la Incera, V.; Manreza Paret, D.; Pérez Martínez, A.; Sanchez, A. Insignificance of the anomalous magnetic moment of charged fermions for the equation of state of a magnetized and dense medium. Phys. Rev. D 2015, 91, 085041.

26. Chatterjee, D.; Elghozi, T.; Novak, J.; Oertel, M. Consistent neutron star models with magnetic field dependent equations of state. Mon. Not. Roy. Astron. Soc. 2015, 447, 3785-3796.

27. Franzon, B.; Dexheimer, V.; Schramm, S. A self-consistent study of magnetic field effects on hybrid stars. Mon. Not. Roy. Astron. Soc. 2016, 456, 2937-2945.

28. Dexheimer, V.; Negreiros, R.; Schramm, S. Hybrid Stars in a Strong Magnetic Field. Eur. Phys. J. A 2012, $48,189$.

29. Alloy, M.D.; Menezes, D.P. Maxwell equation violation by density dependent magnetic fields in neutron stars. Int. J. Mod. Phys. Conf. Ser. 2017, 45, 1760031.

30. Dexheimer, V.; Franzon, B.; Gomes, R.O.; Farias, R.L.S.; Avancini, S.S.; Schramm, S. What is the magnetic field distribution for the equation of state of magnetized neutron stars? Phys. Lett. B 2017, 773, 487-491.

31. Oppenheimer, J.R.; Snyder, H. On Continued Gravitational Contraction. Phys. Rev. 1939, 56, 455-459.

32. Hurley, K.; Dingus, B.L.; Mukherjee, R.; Sreekumar, P.; Kouveliotou, C.; Meegan, C.; Fishman, G.J.; Band, D.; Ford, L.; Bertsch, D.; et al. Detection of a $\gamma$-ray burst of very long duration and very high energy. Nature 1994, 372, 652-654.

33. Galama, T.J.; Vreeswijk, P.M.; van Paradijs, J.; Kouveliotou, C.; Augusteijn, T.; Böhnhardt, H.; Brewer, J.P.; Doublier, V.; Gonzalez, J.F.; Leibundgut, B.; et al. An unusual supernova in the error box of the $\gamma$-ray burst of 25 April 1998. Nature 1998, 395, 670-672.

34. Popov, S.B.; Prokhorov, M.E. Progenitors with enhanced rotation and the origin of magnetars. Mon. Not. R. Astron. Soc. 2006, 367, 732-736.

35. Herrera, L.; di Prisco, A.; Martin, J.; Ospino, J.; Santos, N.O.; Troconis, O. Spherically symmetric dissipative anisotropic fluids: A general study. Phys. Rev. D 2004, 69, 084026.

36. Herrera, L.; di Prisco, A.; Fuenmayor, E.; Troconis, O. Dynamics of Viscous Dissipative Gravitational Collapse: A Full Causal Approach. Int. J. Mod. Phys. D 2009, 18, 129-145.

37. Bonazzola, S.; Gourgoulhon, E.; Salgado, M.; Marck, J.A. Axisymmetric rotating relativistic bodies: A new numerical approach for 'exact' solutions. Astron. Astrophys. 1993, 278, 421-443.

38. Misner, C.; Thorne, K.S.; Wheeler, J.A. Gravitation; Princeton University Press: Princeton, NJ, USA, 1973.

39. Herrera, L.; Di Prisco, A.; Ibáñez, J.; Ospino, J. Axially symmetric static sources: A general framework and some analytical solutions. Phys. Rev. D 2013, 87, 024014.

40. Tolman, R.C. Effect of Inhomogeneity on Cosmological Models. Proc. Natl. Acad. Sci. USA 1934, 20, $169-176$.

41. Oppenheimer, J.R.; Volkoff, G.M. On Massive Neutron Cores. Phys. Rev. 1939, 55, 374-381.

42. Tolman, R.C. Static Solutions of Einstein's Field Equations for Spheres of Fluid. Phys. Rev. 1939, 55, $364-373$.

43. Bondi, H. The contraction of gravitating spheres. Proc. R. Soc. A 1964, 281, 39-48.

44. Bocquet, M.; Bonazzola, S.; Gourgoulhon, E.; Novak, J. Rotating neutron star models with magnetic field. Astron. Astrophys. 1995, 301, 757-775.

45. Cardall, C.Y.; Prakash, M.; Lattimer, J.M. Effects of strong magnetic fields on neutron star structure. Astrophys. J. 2001, 554, 322-339.

46. Frieben, J.; Rezzolla, L. Equilibrium models of relativistic stars with a toroidal magnetic field. Mon. Not. Roy. Astron. Soc. 2012, 427, 3406-3426.

47. Pili, A.G.; Bucciantini, N.; Del Zanna, L. Axisymmetric equilibrium models for magnetized neutron stars in General Relativity under the Conformally Flat Condition. Mon. Not. Roy. Astron. Soc. 2014, 439, 3541-3563. 
48. Gomes, R.O.; Franzon, B.; Dexheimer, V.; Schramm, S. Many-body forces in magnetic neutron stars. Astrophys. J. 2017, 850, 20.

49. Pili, A.G.; Bucciantini, N.; Del Zanna, L. General relativistic models for rotating magnetized neutron stars in conformally flat space-time. Mon. Not. Roy. Astron. Soc. 2017, 470, 2469-2493.

50. Kaplan, D.L.; Bassa, C.; Wang, Z.; Cumming, A.; Kaspi, V.M. Nearby, Thermally Emitting Neutron Stars. AIP Conf. Proc. 2008, 983, 331-339.

51. Haberl, F. AXPs and X-ray dim neutron stars: Recent XMM-Newton and Chandra results. In Proceedings of the 34th COSPAR Scientific Assembly, Houston, TX, USA, 10-19 October 2002.

52. Kaplan, D.L.; van Kerkwijk, M.H. A Coherent Timing Solution for the Nearby Isolated Neutron Star RX J0720.4-3125. Astrophys. J. Lett. 2005, 628, L45-L48.

53. Gill, R.; Heyl, J.S. Statistical ages and the cooling rate of X-ray dim isolated neutron stars. Mon. Not. R. Astron. Soc. 2013, 435, 3243-3250.

54. Kaplan, D.L.; van Kerkwijk, M.H. Constraining the Spin-down of the Nearby Isolated Neutron Star RX J0806.4-4123, and Implications for the Population of Nearby Neutron Stars. Astrophys. J. 2009, 705, 798-808.

55. Kaplan, D.L.; van Kerkwijk, M.H. A Coherent Timing Solution for the Nearby Isolated Neutron Star RX J1308.6+2127/RBS 1223. Astrophys. J. Lett. 2005, 635, L65-L68.

56. Walter, F.M.; Wolk, S.J.; Neuhäuser, R. Discovery of a nearby isolated neutron star. Nature 1996, 379, $233-235$.

57. Van Kerkwijk, M.H.; Kaplan, D.L. Timing the Nearby Isolated Neutron Star RX J1856.5-3754. Astrophys. J. Lett. 2008, 673, L163-L166.

58. Zane, S.; Cropper, M.; Turolla, R.; Zampieri, L.; Chieregato, M.; Drake, J.J.; Treves, A. XMM-Newton Detection of Pulsations and a Spectral Feature in the X-ray Emission of the Isolated Neutron Star 1RXS J214303.7+065419/RBS 1774. Astrophys. J. 2005, 627, 397-403.

59. Kaplan, D.L.; van Kerkwijk, M.H. Constraining the Spin-Down of the Nearby Isolated Neutron Star RX J2143.0+0654. Astrophys. J. Lett. 2009, 692, L62-L66.

60. Van Kerkwijk, M.H.; Kaplan, D.L.; Durant, M.; Kulkarni, S.R.; Paerels, F. A Strong, Broad Absorption Feature in the X-ray Spectrum of the Nearby Neutron Star RX J1605.3+3249. Astrophys. J. 2004, 608, 432-443.

61. Pires, A.M.; Haberl, F.; Zavlin, V.E.; Motch, C.; Zane, S.; Hohle, M.M. XMM-Newton reveals a candidate period for the spin of the "Magnificent Seven" neutron star RX J1605.3+3249. Astron. Astrophys. 2014, 563, A50.

62. Negreiros, R.; Schramm, S.; Weber, F. Impact of Rotation-Driven Particle Repopulation on the Thermal Evolution of Pulsars Phys. Lett. B 2011, 718, 1-5.

63. Negreiros, R.; Schramm, S.; Weber, F. Thermal evolution of neutron stars in two dimensions. Phys. Rev. D 2012, 85, 104019 .

64. De Carvalho, S.M.; Negreiros, R.; Orsaria, M.; Contrera, G.A.; Weber, F.; Spinella, W. Thermal evolution of hybrid stars within the framework of a nonlocal Nambu-Jona-Lasinio model. Phys. Rev. C 2015, 92, 035810.

65. Shternin, P.S.; Yakovlev, D.G.; Heinke, C.O.; Ho, W.C.G.; Patnaude, D.J. Cooling neutron star in the Cassiopeia A supernova remnant: evidence for superfluidity in the core. Mon. Not. R. Astron. Soc. Lett. 2011, 412, L108-L112.

66. Yakovlev, D.G.; Ho, W.C.G.; Shternin, P.S.; Heinke, C.O.; Potekhin, A.Y. Cooling rates of neutron stars and the young neutron star in the Cassiopeia A supernova remnant. Mon. Not. R. Astron. Soc. 2011, 411, 1977-1988.

67. Page, D.; Prakash, M.; Lattimer, J.M.; Steiner, A.W. Rapid Cooling of the Neutron Star in Cassiopeia A Triggered by Neutron Superfluidity in Dense Matter. Phys. Rev. Lett. 2011, 106, 081101.

68. Muslimov, A.; Page, D. Delayed switch-on of pulsars. Astrophys. J. Lett. 1995, 440, L77-L80.

69. Geppert, U.; Page, D.; Zannias, T. Submergence and re-diffusion of the neutron star magnetic field after the supernova. Astron. Astrophys. 1999, 345, 847-854.

70. Viganò, D.; Pons, J.A. Central compact objects and the hidden magnetic field scenario. Mon. Not. R. Astron. Soc. 2012, 425, 2487-2492.

71. Bernal, C.G.; Page, D.; Lee, W.H. Hypercritical Accretion onto a Newborn Neutron Star and Magnetic Field Submergence. Astrophys. J. 2013, 770, 106.

72. Heiselberg, H.; Hjorth-Jensen, M. Phase Transitions in Rotating Neutron Stars. Phys. Rev. Lett. 1998, $80,5485-5488$.

73. Page, D.; Lattimer, J.M.; Prakash, M.; Steiner, A.W. Minimal Cooling of Neutron Stars: A New Paradigm. Astrophys. J. Suppl. Ser. 2004, 155, 623-650. 
74. Kaplan, D.L.; van Kerkwijk, M.H.; Anderson, J. The Parallax and Proper Motion of RX J1856.53754 Revisited. Astrophys. J. 2002, 571, 447-457.

75. Chevalier, R.A. Neutron star accretion in a supernova. Astrophys. J. 1989, 346, 847-859.

76. Bernal, C.G.; Lee, W.H.; Page, D. Hypercritical accretion onto a magnetized neutron star surface: A numerical approach. Revista Mexicana de Astronomía y Astrofísica 2010, 46, 309-322.

77. Muslimov, A.; Page, D. Magnetic and Spin History of Very Young Pulsars. Astrophys. J. 1996, 458, 347.

78. Brown, G.E.; Weingartner, J.C. Accretion onto and radiation from the compact object formed in SN 1987 A. Astrophys. J. 1994, 436, 843-847.

79. Pons, J.A.; Miralles, J.A.; Geppert, U. Magneto-thermal evolution of neutron stars. Astron. Astrophys. 2009, 496, 207-216.

80. Viganò, D.; Rea, N.; Pons, J.A.; Perna, R.; Aguilera, D.N.; Miralles, J.A. Unifying the observational diversity of isolated neutron stars via magneto-thermal evolution models. Mon. Not. R. Astron. Soc. 2013, 434, $123-141$.

81. Gourgouliatos, K.N.; Wood, T.S.; Hollerbach, R. Magnetic field evolution in magnetar crusts through threedimensional simulations. Proc. Natl. Acad. Sci. USA 2016, 113, 3944-3949.

82. Kaspi, V.M. Grand unification of neutron stars. Proc. Natl. Acad. Sci. USA 2010, 107, 7147-7152.

83. Harding, A.K. The neutron star zoo. Front. Phys. 2013, 8, 679-692.

(C) 2018 by the authors. Licensee MDPI, Basel, Switzerland. This article is an open access article distributed under the terms and conditions of the Creative Commons Attribution (CC BY) license (http:/ / creativecommons.org/licenses/by/4.0/). 